$93 \%$ of the isolates $(n=297)$ had MICs of $<0.03 \mathrm{mg} / 1$ which were classified as susceptible (CroS). All CroRed isolates were analysed for mutations in penA (PBP2), mtrR (MtrR), porB (PorB) and ponA (PBP1). 123 (42\%) CroS isolates were randomly selected as coselected Results In total, nine mutation patterns were observed in penA. Patterns I (31.7\%), IX (29.2\%) and XXII (26\%) were most common in the $123 \mathrm{CroS}$ isolates; while patterns IX $(60.8 \%, \mathrm{p}<0.05)$ and XXII $(21.5 \%, p>0.05)$ were predominant in the 23 CroRed isolates. The mosaic penA pattern $\mathrm{X}$ was observed in only one isolate (Cro $\mathrm{MIC}=0.06 \mathrm{mg} / \mathrm{l})$. Seven mutation patterns were noted in $\mathrm{mtrR}$. Among the CroS isolates, $35.8 \%$ had a single mutation (G45D or $\mathrm{A} 39 \mathrm{~T})$ in the DNA binding domain (DBD) and $17.8 \%$ of the isolates carried an A" nucleotide deletion (A-) in the mtr promoter coupled with a G45D mutation. Among the CroRed isolates, $65.2 \%$ carried G45D or A39T single mutations, significantly higher than that of CroS isolates $(\mathrm{p}<0.05)$. The mutation of $\mathrm{A}-/ \mathrm{H} 105 \mathrm{Y}$ was observed in $21.7 \%$ of CroRed isolates as compared to $4 \%$ of CroS isolates $(p<0.05)$. Five mutation patterns at residues G120 and A121 of PorB were observed in the CroRed isolates including double $(47.8 \%$ vs $13 \%$ CroS isolates, $\mathrm{p}<0.05)$ or single $(21.7 \%$ vs $26 \%$ CroS isolates, $\mathrm{p}>0.05)$ mutations. L421P in PBP1 was detected in $87 \%$ CroRed and $13 \%$ CroS isolates, respectively $(p<0.05)$.

Conclusions Mosaic penA pattern X was only observed in one CroRed isolate. The mutations associated with CroRed phenotypes include pattern IX of PBP2, mutations in $\mathrm{DBD}$ and a double mutation of $\mathrm{A}-/ \mathrm{H} 105 \mathrm{Y}$ in $\mathrm{mtrR}$, double mutations in PorB (positions G120 and A121) and L421P of PBP1.

\section{P1-S1.41 EMERGENCE OF AN AFRICAN ANTIMICROBIAL RESISTANCE GENOTYPE IN NEISSERIA GONORRHOEAE STRAINS ISOLATED IN GUANGZHOU, CHINA, 2001-2009}

doi:10.1136/sextrans-2011-050108.41

H P Zheng, B Yang, X Z Wu, J M Huang, W Y Zeng, Y H Xue, H N Jiang, J L Ou. Guangdong Provincial Center for Skin Diseases and STIs Control, Guangzhou, China

Background The continuing spread of drug-resistant gonococci has posed a challenge for successful treatment worldwide. Recently third-generation cephalosporins-resistant strains were isolated in Japan. Guangdong Province in South China has one of the highest gonococcal resistance rates in China and a large number of international migrants. We investigated the invitro antimicrobial susceptibility and genotypes of $N$ gonorrhoeae strains isolated in Guangzhou, the capital city, from 2001 to 2009.

Methods MICs to penicillin, ceftriaxone, tetracycline, ciprofloxacin, and spectinomycin were determined by agar plate dilution and susceptibilities were interpreted according to WHO standards. ßlactamase production was determined by paper acidometric testing. The resistant plasmids were determined for penicillinase-producing $N$ gonorrhoeae (PPNG) and high-level tetracycline resistant $N$ gonorrhoeae (TRNG) by PCR and the isolates were genotyped.

Results Of 1132 consecutive gonococci isolated from 2001 to 2009, no ceftriaxone and spectinomycin resistant strains were found, but the prevalence of strains less susceptible to ceftriaxone rose from $18.2 \%$ to $38.5 \%$. The MIC90 for ceftriaxone showed intermediate sensitivity $(0.06-0.125 \mu \mathrm{g} / \mathrm{ml})$ and spectinomycin near the resistant level $(16-32 \mu \mathrm{g} / \mathrm{ml})$. The resistance to penicillin, tetracycline and ciprofloxacin increased from $81.8 \%, 84.5 \%$ and $72.5 \%$ in 2001 to $90.1 \%, 91.4 \%$ and $98.7 \%$ in 2009 , respectively. A total of $313(27.7 \%)$ PPNG and 486 (42.9\%) TRNG strains were detected. $206(18.2 \%)$ strains were both PPNG and TRNG. PPNG rose from $21.8 \%$ to $29.8 \%$ and TRNG rapidly increased from $27.2 \%$ to $56.3 \%$. Genotyping TEM-1 gene showed 267 (99.6\%) PPNGs in 2001-2008 carried the Asian-type ß-lactamase plasmids and one African-type in 2008. Genotyping of tetM gene showed that all 486 TRNGs were Dutch variants. Conclusion Gonorrhoea resistance continues to be a major public health problem in Guangzhou. The emergence of an African gonor- rhoea resistance variant may be related to the large African diaspora in Guangzhou, migration of Chinese to Africa, or other migration patterns. More research is needed to determine what practices, systems, and behaviours contribute to escalating resistance patterns.

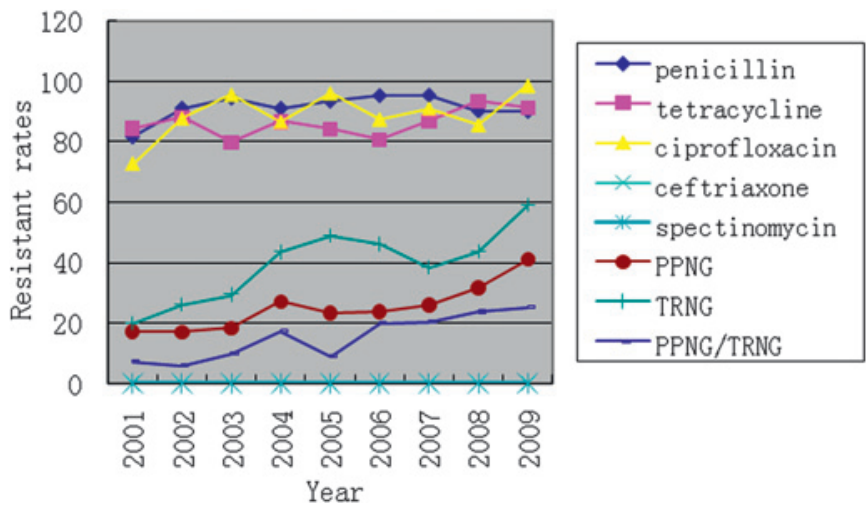

Abstract P1-S1.41 Figure 1 Resistance of $N$ gonorrhoeae isolates in Guangzhou during 2001-2009.

\section{P1-S1.42 RUSSIAN GONOCOCCAL ANTIMICROBIAL SUSCEPTIBILITY PROGRAMME (RU-GASP) - RESISTANCE LEVELS IN 2010 AND TRENDS DURING 2005-2010}

doi:10.1136/sextrans-2011-050108.42

${ }^{1} \mathrm{~N}$ Frigo, ${ }^{2} \mathrm{M}$ Unemo, ${ }^{1} \mathrm{~A}$ Kubanova, ${ }^{1} \mathrm{~A}$ Kubanov, ${ }^{1} \mathrm{~V}$ Solomka, ${ }^{1} \mathrm{~S}$ Polevshikova, ${ }^{3}$ I Lesnaya. 'State Research Centre for Dermatology and Venereology Russian Ministry Health, Russian Federation; ' $S$ wedish Reference Laboratory for Pathogenic Neisseria, Örebro, Sweden; ${ }^{3}$ Polevshikova, Russian Federation

Background Antimicrobial resistance (AMR) in Neisseria gonorrhoeae is a major concern worldwide. AMR surveillance globally is crucial for guiding effective treatment and accordingly for public health purposes. In the former Soviet countries, the knowledge regarding AMR has been limited. However, in 2004 the Russian gonococcal antimicrobial susceptibility programme (RU-GASP), which now acts also under WHO protocols, was initiated. The aims of the present study were to examine and describe the prevalence of $N$ gonorrhoeae AMR in 2010 in Russia, and reveal trends during 2005-2010.

Methods In 2010, $N$ gonorrhoeae isolates (one isolate per patient; $\mathrm{n}=407$ ) collected from 46 surveillance sites distributed in all eight Federal Districts of Russia were examined regarding their susceptibility to ceftriaxone, spectinomycin, azithromycin, penicillin G, ciprofloxacin, and tetracycline using agar dilution method, according to CLSI and WHO protocols. $\beta$-Lactamase production was identified using nitrocefin discs.

Results In 2010, the AMR (intermediate susceptibility) levels were as follows - ceftriaxone $0 \%(0 \%)$, spectinomycin $4.4 \%(12.3 \%)$, azithromycin $4.9 \%(10.4 \%)$, penicillin G $32.5 \%$ (39.9\%), ciprofloxacin $53.2 \%$ (3.0\%), and tetracycline $41.9 \%$ (26.6\%). Three isolates $(0.7 \%)$ were resistant to all antimicrobials except ceftriaxone. During 2005-2010, the AMR has remained high to penicillin G, tetracycline, and ciprofloxacin. The proportion of resistant isolates to spectinomycin and azithromycin has ranged from $0 \%(2005)$ to $7.2 \%$ (2008), and $0.4 \%$ (2008) to $4.9 \%$ (2010), respectively. All isolates in 2005-2010 were susceptible to ceftriaxone. Nevertheless, the minimum inhibitory concentration (MIC) distribution of ceftriaxone has rapidly shifted to higher MICs, and isolates at the breakpoint for intermediate susceptibility/resistance $(0.25 \mathrm{mg} / \mathrm{l} ; \mathrm{n}=8[2 \%]$ in 2010$)$ have rapidly increased over the years. Conclusions The AMR of $N$ gonorrhoeae in Russia is high and ceftriaxone ( ${ }^{3} 250 \mathrm{mg}$ ) should be first-line for empirical treatment. If there are no access to ceftriaxone or in the presence of severe $\beta$ lactam antimicrobial allergy, spectinomycin is recommended for use. 